\title{
A token-based dynamic scheduled MAC protocol for health monitoring
}

\author{
Xiaoming Yuan ${ }^{1}$, Changle Li ${ }^{*}$, Li Yang ${ }^{1}$, Wenwei Yue ${ }^{1}$, Beibei Zhang ${ }^{1}$ and Sana Ullah ${ }^{2,3}$
}

\begin{abstract}
Developments of wireless body area networks (WBANs) facilitate the pervasive health monitoring with mHealth applications. WBANs can support continuous health monitoring for the human body in convenience and high efficiency without any intervention. The monitoring data in health care have the characteristics of various data flows and heterogeneous data arrival rates, the transmission of which must be in timeliness and reliability, especially the burst data. Moreover, the energy-constraint nodes should be provident in energy consumption. Designing MAC protocols with high reliability and energy efficiency for WBANs is the prime consideration. In this paper, we propose a token-based two-round reservation MAC (TTR MAC) protocol based on IEEE 802.15.6 with considering the data features of health monitoring. With analyzing the characteristics of monitoring data, one-round reservation is conducted for periodic data and two-round reservation is generated adaptively for burst data to save energy. Besides, TTR MAC protocol assigns appropriate number of allocation slots to nodes in heterogeneous data arrival rates. Furthermore, a token is introduced on the basis of user priority and health severity index to indicate the transmission order of nodes with burst data, which highly decreases the average delay. In addition, a bit sequence scheduled algorithm is proposed for m-periodic $(m>1)$ monitoring data for network capacity expansion. The simulation results show that TTR MAC protocol achieves higher energy efficiency and longer lifetime compared with IEEE 802.15 .6 and other one-round reservation MAC (OR MAC) protocols for both 1-periodic and $m$-periodic data.
\end{abstract}

Keywords: WBAN, IEEE 802.15.6, MAC, Scheduled mechanism, Energy efficiency, Health monitoring

\section{Introduction}

The recent proliferation of wireless communication technologies and wearable medical devices has motivated the revolution in the area of mobile health, referred to by the term "mHealth" $[1,2]$. The mHealth field has emerged as a sub-segment of electronic health (eHealth) [3]. mHeath encompasses the use of mobile devices in collecting community and patient-level health data, real-time monitoring of patient vitals, and delivery of health-care information to practitioners, researchers, and patients. Health monitoring records the change of human body in physiology, behavior, and daily environment. The monitoring data have the potential to yield new insights in disease inducements and to be used to assist diagnosis and prescription for both healthy people and patients.

*Correspondence: clli@mail.xidian.edu.cn

1 State Key Laboratory of Integrated Services Networks, Xidian University, No.2 Tai Bai South Road, 710071 Xi'an, China

Full list of author information is available at the end of the article
Wireless body area network (WBAN) is a short-range wireless communication network with several sensor nodes attached to or implanted in a human body. The WBAN can provide continuous and reliable health monitoring with great freedom and convenience. WBAN promotes the successful development of mHealth system and health-care applications. A health monitoring system is presented in Fig. 1. Sensor nodes in WBANs collect the biomedical information of human body, such as body temperature, heartbeat, and blood pressure in medical applications, and propagate their records back to the hub or the base station. The hub then processes and analyzes these data to give the correct suggestion to patients or transmits these data to the remote health-care center to call for more assistance. Moreover, WBAN has significant potential for applications in military and sports training, interactive gaming, personal information sharing, and secure authentication $[4,5]$. It can be seen that 


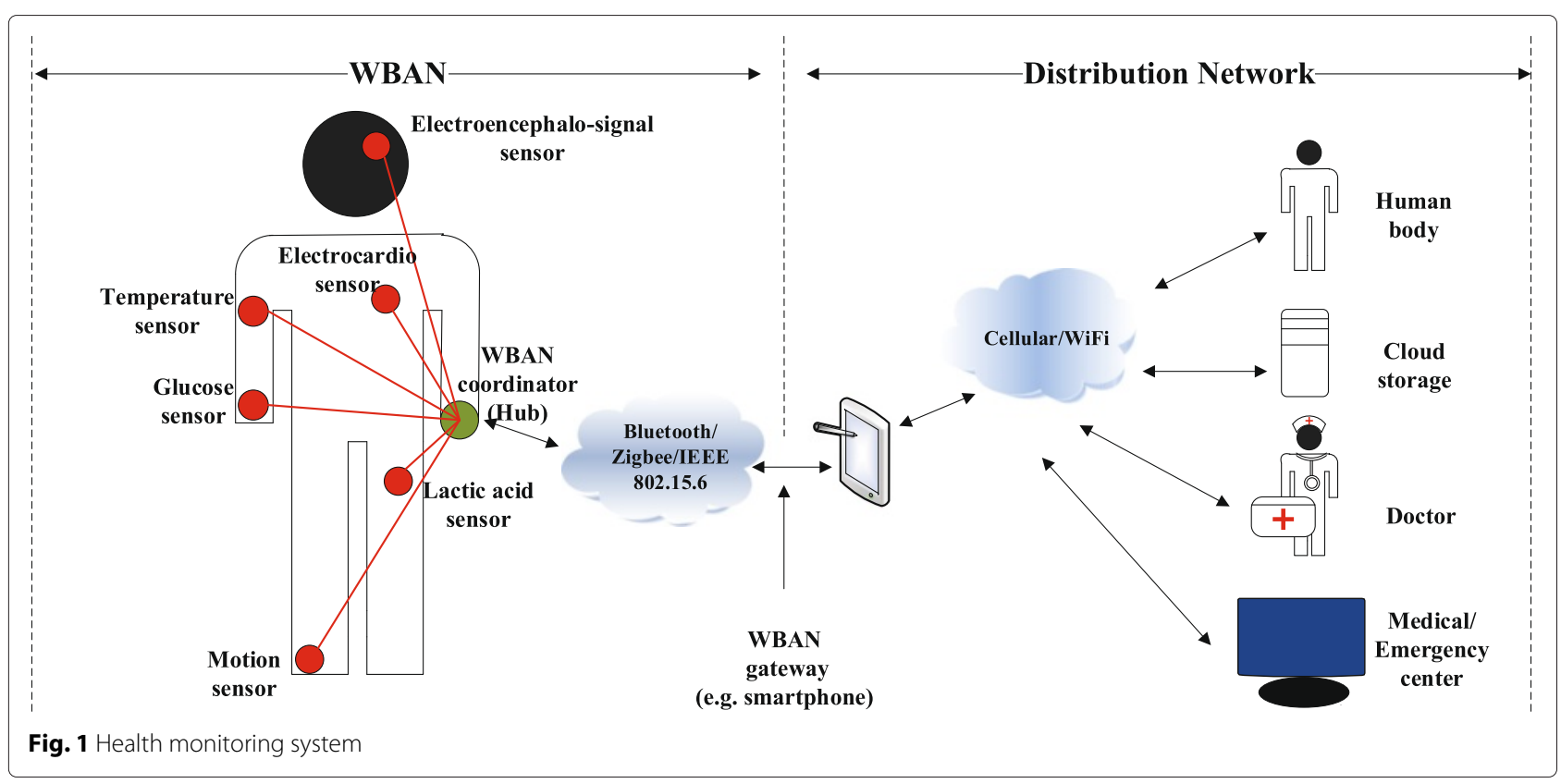

WBANs can offer a great solution to health monitoring in mHealth owing to its wide range of utility in various application scenarios [6-8]. An overview of different technical solutions to the challenges of mHealth applications for WBANs is presented in [8].

There are many technologies and design challenges in WBAN for health monitoring [9-11]. On one hand, the survival time of sensor nodes that rely on batteries as energy supplies are expected to sustain at least a few months or years, especially for implanted sensor nodes. Sensor nodes in WBAN should be stringent in energy consumption. On the other hand, the life-threatening data collected from sensor nodes should be transmitted in reliability and timeliness because the life-threatening data would make sense only when they are transmitted timely. The medium access control (MAC) layer plays a pivotal role to guarantee the energy-saving and real-time data transmissions in the health monitoring system. Energyefficient and reliable MAC protocols are appropriate solutions to the aforementioned challenges.

There have been a large number of MAC protocols and algorithms proposed to increase energy efficiency and network reliability for wireless sensor networks (WSNs). However, such techniques cannot be used effectively for WBAN. Since WBAN has distinctive human-centered characteristic that is different from conventional WSN $[5,12]$. Besides, few previous studies have considered the variant characteristics of data in WBAN for health monitoring; thus, it is hard to achieve optimal performance if the existing works are directly used for health monitoring. The characteristics of data in WBAN for health monitoring are reflected in the following three aspects:
1. Different data arrival patterns under various scenarios: Data in routine health monitoring are generated periodically where the data arrival rate can be determined by the sampling rate uniquely. But when the collected data exceed the safe range, sensor nodes generate burst data or emergency data that should be processed timely. What's more, additional data are collected to improve the accuracy of burst traffic. The data arrival pattern is hard to predict.

2. Heterogeneous data arrival rates: Disparate sensor nodes are different in traffic varieties. Sampling text information, images, and video information are in different arrival rates for sensors. For example, electrocardiogram data rate is multiple times higher in comparison with body temperature.

3. Correlation between sensor nodes: Sensors in health monitoring are correlated with each other. A patient suffering from a fever will trigger the changes in temperature, blood pressure, and respiration sensors at the same time [13]. If all the related data are packed or clumped together to be transmitted in disorder, the strongest correlation data of vital signs to the illness may endure a delay and make no sense in the worst-case scenario.

In this paper, we propose a MAC protocol named tokenbased two-round reservation MAC (TTR MAC) based on IEEE 802.15.6 for health monitoring in WBAN. IEEE 802.15.6 [14] is an international standardization specifically designed to support WBANs. Different from IEEE 802.15.4 [15], IEEE 802.15.6 specifies the nodes in WBAN into eight user priorities, which guarantees the quality of 
service $(\mathrm{Q} o \mathrm{~S})$ of nodes with higher priority. It provides powerful foundation for WBAN designers with comprehensive technical guidance in MAC and physical (PHY) layer. The general monitoring data in WBAN are periodic. Scheduled-based MAC protocols satisfy the data transmission requirements and provide good solutions. Burst data are critical in severe condition, the transmissions of which should be timely and reliable. However, IEEE 802.15.6 will harvest the optimal performance only when appropriate handle mechanism and network parameters are designed based on the specific application scenario. Therefore, we propose the TTR MAC protocol based on the IEEE 802.15.6, taking the user priorities (UPs) and critical conditions of data into consideration. The contributions of this paper are presented below:

- TTR MAC discriminates the monitoring data to periodic data and burst data. It conducts two-round reservations to guarantee the data transmissions for the two data types to reduce energy consumption and overhead induced by control frames.

- We build a relationship between the appropriate number of allocation slots and heterogeneous data arrival rates, which helps to utilize the time resource more efficiently and save more energy.

- In TTR MAC protocol, the hub uses a token to assign additional allocation intervals for burst data according to the user priority of nodes and the criticality of burst data. Because the correlation between sensor nodes may generate several burst data streams at the same time. In this way, TTR MAC protocol can guarantee the most emergency data to be transmitted effectively and timely.

- A bit sequence-scheduled mechanism is designed for $m$-periodic $(m>1)$ data, which expands the network capacity and better satisfies the QoS of nodes.

The rest of paper is organized as follows. Section 2 discusses the related work including the motivation behind our work and the IEEE 802.15.6 standard. Section 3 presents the details of the proposed TTR MAC protocol. Section 4 introduces the bit sequence algorithm specific to $m$-period data. The performance evaluation is discussed in Section 5 while Section 6 concludes the paper and drives the future works.

\section{Related work}

\subsection{Motivation}

A WBAN is expected to operate a long period with minimum energy consumption of sensor nodes. Ascribing the ubiquitous human-centered characteristic of WBAN, the data transmissions have more stringent requirements, for example, timely response to emergency data with minimum delay, minimum overhead in the network, and minimum number of data collision and retransmission times to save energy. On the average, MAC protocols are designed to maximize network lifetime via reducing the control packet overhead, the energy dissipation in collision and retransmission, and idle listening and overhearing. It follows that energy-efficient and reliable MAC protocols in the minimum overhead without interference are reasonable solutions to these requirements.

It is also our objective to address the aforementioned issues. Besides, according to the characteristics of data in health monitoring that scheduled access and improvised access are needed simultaneously, our proposed MAC aims to answer these questions in health monitoring:

- How to transmit the periodic and burst data generated in health monitoring effectively?

- How to assign appropriate allocation intervals for the sensor nodes with diverging data arrival rates?

- How to guarantee lower delay for nodes with high user priority and critical conditions when several burst data streams are generated due to the correlation between sensor nodes?

MAC protocols are mostly contention-based, scheduled-based, and hybrid-based. A survey of MAC protocols for WBAN is studied in [16]. Contentionbased MAC protocols such as carrier sense multiple access/collision avoidance (CSMA/CA) protocols have no strict time synchronization constraint which makes it scalable and easy to deploy, such as typical T-MAC [17], WiseMAC [18], and S-MAC [19]. The performance of IEEE 802.15.6 CSMA/CA focusing on throughput and delay is introduced in [20]. The CSMA/CA mechanism of IEEE 802.15.6 under non-saturation regime and saturation condition is introduced in [21] and [22], respectively. However, contention-based MAC protocols suffer some flaws when designed for WBANs: idle listening, overhearing, and frequency collisions cost too much unnecessary energy, and the network may endure performance deterioration. Though some proposed energy-harvesting MAC protocols such as [23] following the CSMA/CA rules that allow relay nodes to charge their batteries, sometimes battery recharge is difficult and the energy efficiency of MAC protocol is the prime consideration.

The deployment of health monitoring shows that the hub has relatively rich resources to schedule the process of communication with nodes, thus scheduled-based MAC protocols [24-27] provide better solutions for WBAN. Reference [24] studies the ultra-low power time division multiple access (TDMA) MAC protocols based on IEEE 802.15.4. References $[25,26]$ pay their attention to wakeup mechanism to reduce energy consumption and prolong the network lifetime in WBAN. S-TDMA [27] and PEH-QoS $[28,29]$ applies a scheduled scheme based on 
IEEE 802.15.6 for energy efficiency and energy harvesting for WBAN, respectively. Besides, a cloud-assisted random linear network coding MAC (CLNC-MAC) protocol on IEEE 802.15.6-based schedule scheme focusing on relay WBAN network is studied in [30]. Whereas, they do not take the different user priorities and critical condition of emergency data into consideration, some other efficient scheduled allocations reduce the duty cycle of sensor nodes and their power consumption, such as [31] and [32], but they failed in adapting the heterogeneous data arrival rates in WBANs. In [33], they conquer the heterogeneous data arrival rates at the premise that every node in WBAN can communicate with each other, which increases the operation complexity of the nodes in realistic situation and leads to more energy consumption.

Most of the hybrid-based MAC protocols [34-38] designed for WBAN request allocation in the contention periods and assign allocation in the contention-free periods to satisfy the diverse demands for different nodes. References $[35,36]$ design traffic adaptive MAC protocols for WBAN that request frame reservation in CSMA and data transmission in TDMA. The superframe is divided into downlink and uplink frames in [37]. The uplink frame is further subdivided into a contention-access period and contention-free period. E. Ibarra et al. [38] exploit energy harvesting with considering energy level of sensor nodes. Nodes with high energy level enjoy a reserved polling access phase while nodes with low energy level employ a random access phase in [38]. But it should be noticed that they notify traffic information in each superframe, which makes a huge overhead that will deteriorate the performance in WBANs.

We design a TTR MAC protocol that employs scheduled mechanism to satisfy the data transmission requirements and that provides good solutions to WBAN. The TTR MAC analyzes the characteristics of monitoring data in WBAN and designs two kinds of reservation modes for periodic and burst data. The one-round reservation for periodic data is conducted once nodes join in the network, and appropriate allocation intervals for each node are assigned. A token-based two-round reservation will generate if there are burst data in the network with both user priority and critical condition considered. The design increases the adaptivity of scheduled mechanism and guarantees low delay for nodes with higher priority and critical conditions. The specific mechanism and performance analysis of the proposed TTR MAC are introduced in Section 4. Moreover, if several $m$-periodic sensor nodes are assigned into a superframe, the traffic distribution is unbalanced, which will lead a deterioration in the network performance. We present a bit sequence scheme that the nodes can share the slots with same number in different superframes to increase the resource utility and network capacity for $m$-periodic data to address the aforementioned issue.

\subsection{IEEE 802.15.6 overview}

IEEE 802.15.6 [14] is a standard for short-range, wireless communications in the vicinity of, or inside, a human body. IEEE 802.15.6 supports for the QoS with low power and minimizes the specific absorption rate (SAR) into the body, making it an appropriate solution for WBANs.

In IEEE 802.15.6, all nodes and hubs should establish a time reference if their medium access is scheduled in time. Time base is divided into equal length beacon periods (superframes), and each superframe is further divided into equal length allocation slots, which is numbered from $0,1, \ldots, x$, where $x \leq 255$, as shown in Fig. 2. Allocation interval for specific node may span more than one allocation slot and can be expressed by the number of slots at the start and end.

In order to support the diverse application scenarios in WBANs, IEEE 802.15.6 operates in one of the following three access modes: beacon mode with superframes, non-beacon mode with superframes, and non-beacon mode without superframes. In the beacon mode with superframes, a beacon is transmitted at the start of each superframe to declare the information related to BAN identification, synchronization, and superframe structure.

The superframe structure under beacon mode with superframes is shown in Fig. 3. The superframe consists of seven periods: two exclusive access phases (EAPs), two random access phases (RAPs), two managed access phases (MAPs), and a contention access phase (CAP). Nodes utilize contended scheme-CSMA/CA or slotted Aloha to access channel in EAP, RAP, and CAP, while

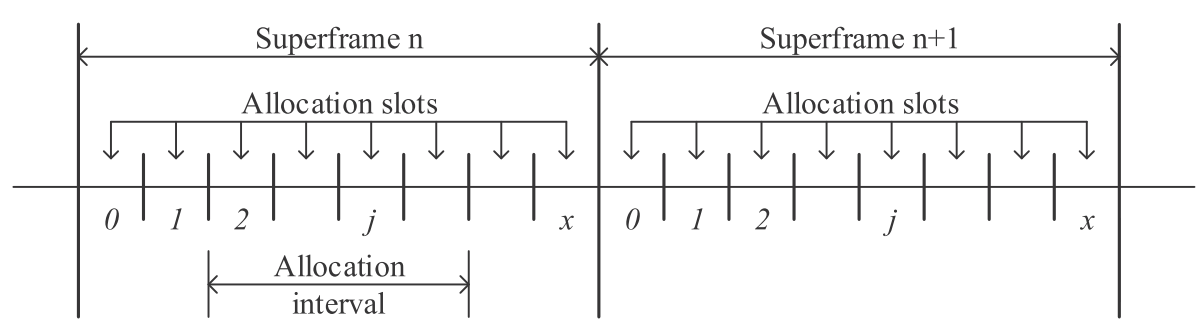

Fig. 2 Time reference base in IEEE 802.15.6 


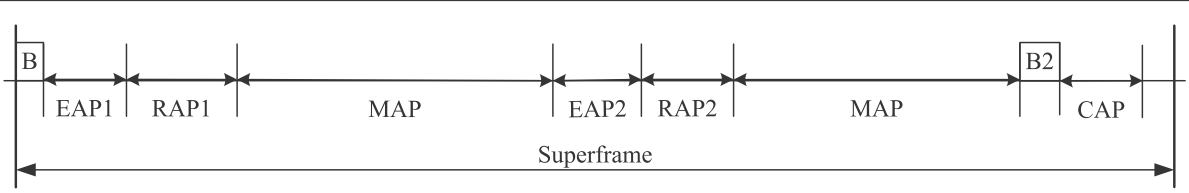

Fig. 3 Superframe structure in beacon mode with superframes in IEEE 802.15.6

MAP supports scheduled access, unscheduled access, and improvised access. It should be noticed that EAP is only used for the highest priority (totally eight defined UPs) data such as emergency events while there is no traffic variety limits in RAP and CAP.

Scheduled access supports either 1-periodic or $m$ periodic $(m>1)$ allocations. Nodes communicate with the hub in each superframe in 1-periodic scheduled allocation and in every $m$ superframes in $m$-periodic scheduled access. Unscheduled access supports 1-periodic, $m$-periodic, or round robin allocations. Improvised access is an independent access method to send poll or post to guarantee an immediate or future allocation interval for a specific node. It can be utilized as the supplemental access method to scheduled access and unscheduled access.

\section{Proposed scheme}

In this section, we will present the proposed TTR MAC in details. We classify the data in health monitoring into periodic data and burst data and conduct two-round reservations to handle these two types of data, respectively. In order to allocate flexible time slots for nodes with various data arrival rates, we build a relationship between the appropriate number of allocation slots and data arrival rates.

\subsection{Data classification}

Upon considering the data characteristics in health monitoring, various data flows, heterogeneous data arrival rates, and correlation between sensor nodes, TTR MAC classifies the data into periodic data and burst data. The periodic data are in charge of routine monitoring. They report the changes in biomedical signals at a constant frequency. However, when the monitoring data exceeds the predefined safe range, nodes should collect additional data to record the variation more accurately which is hard to predict. And due to the correlation between sensor nodes, there will be several data streams occurring simultaneously thus, we call these additional data as the burst data. The hub can know the arrival rates of the periodic data from the nodes based on their sampling rate as long as the nodes join in the network. The arrival rate of burst data is hard to predict; thus, it can only be informed with real-time communication.

\subsection{Two-round reservation}

\subsubsection{Superframe structure}

The superframe structure of TTR MAC is shown in Fig. 4. It starts with a beacon followed by the first-round reservation period (FRRP) and the second-round reservation period (SRRP) and ends with the sleep period (SP). The FRRP aims to transmit the periodic data and raise the second-round reservation if burst data occurs. The SRRP takes charge of the burst data transmission. In addition, all devices, including nodes and hub, enter the sleep period to save energy when there are no data transmissions.

\subsubsection{First-round reservation}

The first-round reservation is designed for the periodic data. Once a node joins in a specific WBAN, its periodic data arrival rate can be determined by its sampling rate. The first-round reservation takes place in the connection procedure when a node joins the WBAN. The connection request frame piggybacks the related information for reservation. The reservation procedure is depicted in Fig. 5.

Before transmitting sensor data to the hub, a node should make a connection with the hub to join in the network. Thus, it will send a connection request frame to the hub with the CSMA/CA scheme due to having no

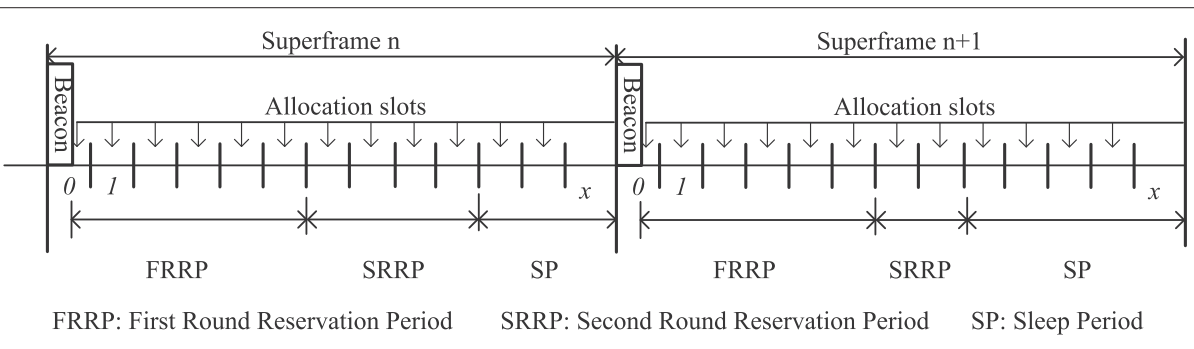

Fig. 4 Superframe structure of TTR MAC 


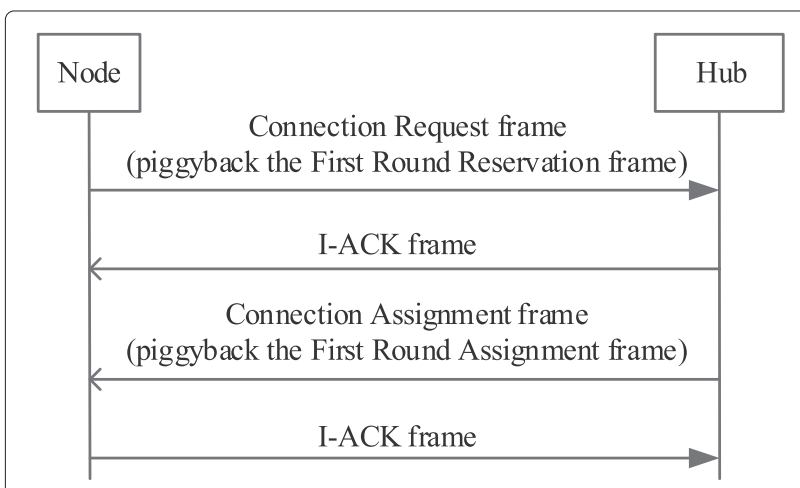

Fig. 5 The first-round reservation procedure

information about the scheduled allocation yet. In order to obtain the appropriate number of allocation slots and utilize the time resource effectively, nodes insert its periodic data arrival rate and data cycle into the connection request frame and transmit it to the hub. Then, the node waits the immediate acknowledgement (I-ACK) or the connection assignment frame from the hub. If neither of the two frames is received by node within the predefined time, it will retransmit the connection request frame. The specific frame formats of connection request and connection assignment frame are depicted in Fig. 6.

After receiving the connection request frame successfully, the hub checks its available resource and evaluates whether to accept the node accessing the network. If the remaining resource is enough to access the node in, the hub extracts the node's periodic data arrival rate. While considering the packet processing in MAC and PHY layer and the transmission characteristics of wireless channel, TTR MAC builds a relationship between the appropriate number of allocation slots and data arrival rate to help the hub assigns allocation slots for the specific node.
Since all nodes and hub have established a time reference base and all slots are numbered in order, the hub can determine the interval start and interval end for a specific node, which makes up the first-round allocation assignment frame, as shown in Fig. 7. For example, when the hub receives the allocation request from node $\mathrm{A}$, it makes $S_{A}$ be the least number of slot which not be used and $E_{A}=S_{A}+N_{\text {AllocationSlot }}-1$, which means the allocation for node A starts at the beginning of the allocation slot numbered $S_{A}$ and ends at the end of the allocation slot numbered $E_{A}$.

Then, the hub piggybacks this assignment frame into the connection assignment frame. If the hub receives the expected ACK, it records the assigned slot number from $S_{i}$ to $E_{i}$ in order to receive data from node $i$ when this interval comes. Otherwise, the hub retransmit the connection assignment frame once the predefined time expires.

When the node receives the connection assignment frame successfully, it extracts the allocation interval information and replies I-ACK to the hub. Hereto, the node has built a connection with the hub and obtained appropriate number of allocation slots to transmit its periodic data.

Upon finishing the first-round reservation for each node, the hub transmits beacon frame to start the data transmission. When the allocated interval commences, each node transmits the buffered data to the hub. The hub receives the uplink data from each node in each allocation interval. All nodes and hub turn into the sleep mode in the rest of the time to save energy.

The first-round reservation is only conducted when the node joins the network and is along with the connection procedure through asserting the necessary information about periodic data into the connection frame. In this way, TTR MAC executes the first-round reservation with a little overhead, which will save many unnecessary energy.

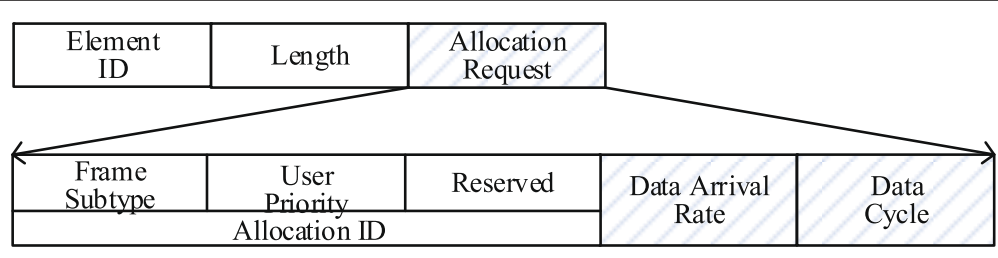

(a)

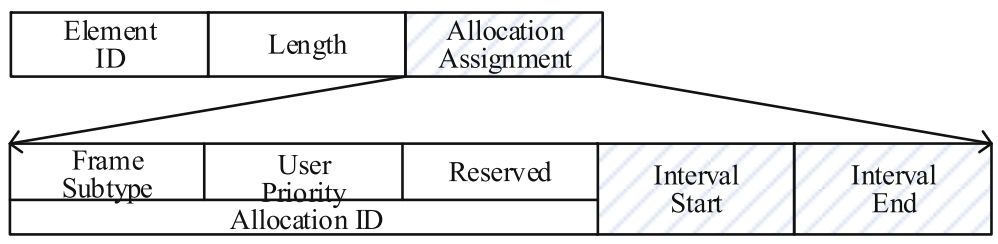

(b)

Fig. 6 a Connection request frame for the first-round reservation. b Connection assignment frame for the first-round reservation 


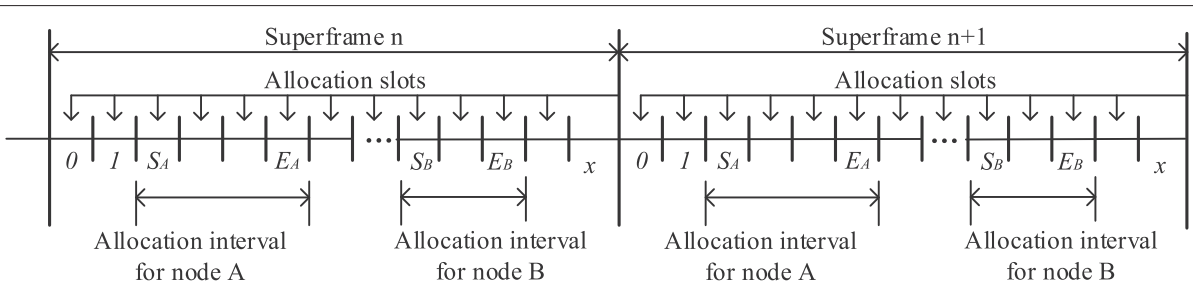

Fig. 7 Allocation assignment for node $A$ and node $B$

On the other hand, TTR MAC cannot adapt well in the monitoring scenario where one node has dynamic data arrival rates, i.e., dynamic sampling rates at different times. Since the first-round reservation frame is only transmitted during the connection procedure, the hub assigns appropriate allocation slots for the node according to the original data arrival rate. After building the connection with the hub, though the data arrival rate changes, the number of allocation slots cannot get adjustment timely. So, TTR MAC is only appropriate to use in the routine health monitoring scenario with relatively fixed sampling rate.

\subsubsection{Second-round reservation}

The first-round reservation assigns appropriate allocation slots for periodic data of different nodes. However, if the monitoring data exceed the safe range, the node needs to sample additional data to record the variation more accurately, which means additional allocation slots should be assigned for these data. What's more, there may be several burst data streams emerging at the same time due to the correlation among sensor nodes in health monitoring. Thus, the second-round reservation period should both handle the burst streams from several sensors and guarantee lower delay for nodes with higher user priority and severer conditions.

In order to obtain additional allocation slots for burst data, nodes with burst data should transmit the secondround allocation request frame to the hub in the allocation interval assigned in the first-round reservation. The format of the second-round allocation request frame is designed as shown in Fig. 8. Considering that the burst data are mostly from nodes in severe condition, TTR MAC should guarantee a lower delay for the severer node.

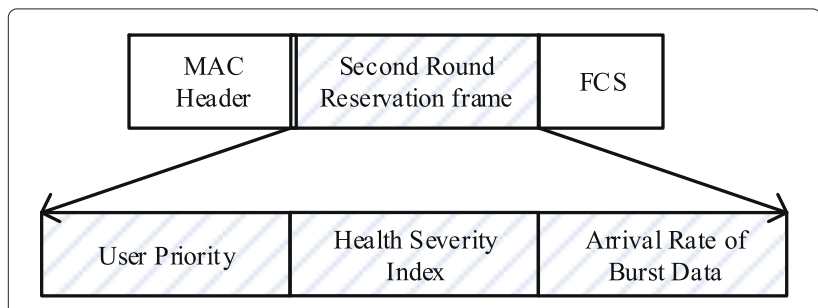

Fig. 8 Second-round allocation request frame
It means that the severer node should be assigned to the slots earlier. So the user priority, health severity index, and arrival rate of burst data are included in the request frame.

(a) User priority (UP) IEEE 802.15.6 assigns different UPs for different frame types, which vary from UP0 to UP7. The UP is determined based on the data type generated by nodes, such as background data, video data, and emergency reports. The higher UP of a node is, the greater privilege to access the medium it has. UP7 is the highest while UP0 is the lowest. The predefined relationship of prioritized access can be attained in Table 1.

(b) Health severity index $(H)$

Health severity index denotes the degree of data deviating from the safety range, which is calculated by Eq. (1).

$$
H= \begin{cases}\min \left(\frac{\theta_{l}-\theta}{\theta_{u}-\theta_{l}}, 1\right) & \theta<\theta_{l} \\ 0 & \theta_{l} \leq \theta \leq \theta_{u} \\ \min \left(\frac{\theta-\theta_{u}}{\theta_{u}-\theta_{l}}, 1\right) & \theta>\theta_{u}\end{cases}
$$

where $\theta$ denotes the value of collected data, $\theta_{l}$ denotes the lower bound, and $\theta_{u}$ is the upper bound of the safe range. It can be seen that $0 \leq H \leq 1$. The higher $H$ is, the severer condition node is in.

(c) Volume of burst data $N_{\text {BurstData }}$ Nodes estimate the volume of burst data in buffer in order to provide a reference for the hub to assign

Table 1 User priorities representation

\begin{tabular}{lll}
\hline User priorities & Traffic designation & Frame type \\
\hline 0 & Background & Data \\
1 & Best effort & Data \\
2 & Excellent effort & Data \\
3 & Video & Data \\
4 & Voice & Data or management \\
5 & Medical data or network control & Data or management \\
6 & High priority medical data & Data or management \\
7 & or network control & \\
& Emergency or medical implanted & Data \\
\hline
\end{tabular}


additional allocation slots for them. When the hub receives the second-round allocation request frame, the hub replies ACK with the added time field to inform the nodes to receive the second-round allocation assignment frame. Then, the hub will introduce the token to determine the transmission order of nodes with burst data. The token is calculated by considering both the node's user priority and the critical condition.

$$
\begin{array}{r}
\text { token }=\rho_{1} \times \frac{\mathrm{UP}}{8}+\rho_{2} \times H \\
\text { s.t. } 0 \leq \rho_{1}, \rho_{2} \leq 1, \rho_{1}+\rho_{2}=1
\end{array}
$$

where $\rho_{1}$ is the weight factor of the user priority while $\rho_{2}$ is the weight factor of the critical condition to attain the token value.

Nodes with the higher token are awarded with the higher privilege to transmit, which guarantees a better quality of service for the urgent data. Then, the hub assigns additional allocation slots for the burst data and writes the allocation interval in the second-round allocation assignment frame. It should also be emphasized that if the remaining allocation slots are insufficient to transmit all the burst data, the hub will deny to assign allocation slots for nodes with lower token.

If the sensor nodes in health monitoring are sensitive to security, the hub will transmit the second-round allocation assignment frame in unicast manner, if not, in broadcast manner. The format of the second-round allocation assignment frame is the same as the first-round allocation assignment frame, as shown in Fig. 6b. The difference is that there may be several allocation assignment frames following the length field in broadcast manner, each of which dedicates the start and the end of allocation intervals for the specific node.
Upon receiving the second-round allocation assignment frame, nodes check whether the hub assigns additional slots for it or not. If it has assigned, the nodes will transmit the burst data when the specific allocation interval commences. Otherwise, it will wait for the next superframe to retry the second-round reservation.

It should be noticed that the second-round reservation only takes place when node has burst data. If there is no burst data, all nodes and hub enter the sleep period to save energy.

\subsubsection{An example of TTR MAC}

In order to illustrate the operation in TTR MAC clearly, we draw a simple example as shown in Fig. 9. There are three nodes in health monitoring: node $\mathrm{A}$, node $\mathrm{B}$, and node $C$. Assuming that the three nodes have connected into the network, which means that they have informed their periodic data arrival rate to the hub, and the hub has assigned appropriate number of allocation slots for their periodic data through the first-round reservation. In the $i$ th superframe, both node $\mathrm{A}$ and node $\mathrm{C}$ have burst data in buffer; thus, they raise the second-round reservation in their first allocation interval. Upon receiving the second-round allocation request frame, the hub determines the transmit order and the number of allocation slots for burst data of these two nodes. In this example, we assume that node $C$ has higher token, which means the data of node $\mathrm{C}$ are more severer than node $\mathrm{A}$. Thus, node $\mathrm{C}$ conducts transmission prior to node $\mathrm{A}$. Due to no burst data, node $\mathrm{B}$ does not conduct the second-round reservation and enters sleep mode after finishing its data transmission.

\subsection{Appropriate number of allocation slots}

Since sensor nodes in health monitoring own heterogeneous data arrival rates, it is necessary to assign the

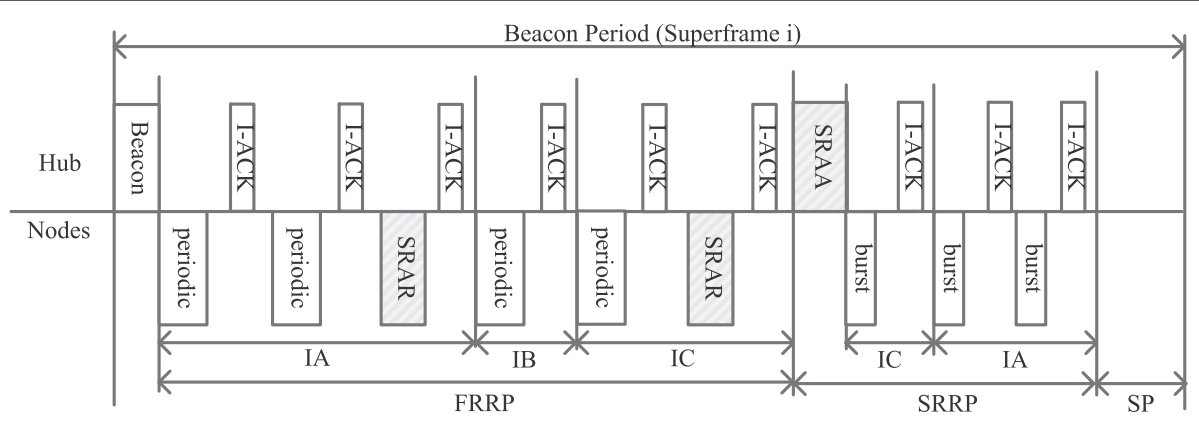

SRAR: Second Round Allocation Request frame SRAA: Second Round Allocation Assignment frame
IA: Interval for Node A
IB: Interval for Node B
IC: Interval for Node C

Fig. 9 A simple example of TTR MAC 
appropriate allocation intervals for each node according to their data arrival rates. Dynamic and flexible allocation scheme will increase channel utilization and help the network adapt better with heterogeneous sensor nodes. Therefore, TTR MAC builds a relationship between the appropriate number of allocation slots and data arrival rate based on the operation in MAC and PHY in IEEE 802.15.6.

\subsubsection{Allocation interval for nodes with specific data arrival rate}

Firstly, the hub calculates the total time needed to transmit the data in a superframe with arrival rate $\lambda_{i}$, which is called allocation interval $t_{\text {interval }}$. The $t_{\text {interval }}$ includes the time duration to transmit all packets, interframe spacing, time duration of ACK packet, guard time, and propagation delay time.

$$
\begin{aligned}
t_{\text {interval }}= & (\text { num }-1) \times t_{\text {packet }}+t_{\text {RemainPacket }}+\text { num } \\
& \times\left(\mathrm{pSIFS}+t_{\mathrm{ACK}}\right)+\mathrm{pSIFS}+\mathrm{GT}+t_{\text {prodelay }} .
\end{aligned}
$$

num denotes the number of packets into which data are fragmented. With the length of the MAC frame body $N_{\text {MACFrameBody }}$ in bit, the number of packets for 1-periodic node with specific data arrival rate is

$$
\text { num }=\left\lceil\frac{\lambda_{i} \times t_{\text {superframe }}}{N_{\text {MACFrameBody }}}\right\rceil,
$$

where $\lambda_{i}$ denotes the data arrival rate for specific node and $t_{\text {superframe }}$ denotes the time duration of each superframe. The number of packets for $m$-periodic node can be obtained by

$$
\text { num }=\left\lceil\frac{\lambda_{i} \times t_{\text {superframe }} \times m}{N_{\text {MACFrameBody }}}\right\rceil
$$

$t_{\text {packet }}$ represents an integrity packet duration. The structure of the physical layer protocol data unit (PPDU) defined by IEEE 802.15.6 is depicted in Fig. 10. According to the PHY technologies demonstrated in IEEE 802.15.6, the total duration $t_{\text {packet }}$ of a packet is

$$
t_{\text {packet }}=\frac{N_{\text {preamble }}+N_{\text {header }} \times S_{\text {header }}+\frac{N_{\text {total }} \times S_{\text {PSDU }}}{\log _{2} M}}{\text { Symbol rate }},
$$

where $S_{\text {header }}$ denotes the spreading factor for the physical layer convergence protocol (PLCP) header and $S_{\text {PSDU }}$ denotes the spreading factor for the transmission mode. $M$ is the modulation constellation size for the transmission mode. The $N_{\text {total }}$ is the total bits which flow in the PHY, and $N_{\text {PSDU }}$ stands for the length of the physical service data unit (PSDU) when the MAC frame is packed with the MAC header and FCS

$$
N_{\text {total }}=N_{\mathrm{PSDU}}+N_{\mathrm{CW}} \times(n-k)+N_{\text {pad }}
$$

$N_{\mathrm{PSDU}}=\left(N_{\mathrm{MACH}}\right.$ eader $\left.+N_{\mathrm{MACFrameBody}}+N_{\mathrm{FCS}}\right) \times 8,(8)$

where $N_{\mathrm{CW}}$ denotes the number of Bose, Ray-Chaudhuri, Hocquenghem code $(\mathrm{BCH})$ codewords and $N_{\text {pad }}$ is the number of pad bits, which shall be appended after the $\mathrm{BCH}$ encoder to align the bit stream on a symbol boundary and can be determined by the correlated parameters in IEEE 802.15.6. They can be calculated by Eqs. (9) and (10), where $n$ and $k$ are selected by BCH code. Different packet components and modulation modes may employ different code rates.

$$
\begin{aligned}
N_{\mathrm{CW}}= & \left\lceil\frac{N_{\mathrm{PSDU}}}{k}\right\rceil \\
N_{\mathrm{pad}}= & \log _{2}(M) \times\left\lceil\frac{N_{\mathrm{PSDU}}+N_{\mathrm{CW}} \times(n-k)}{\log _{2}(M)}\right\rceil \\
& -\left[N_{\mathrm{PSDU}}+N_{\mathrm{CW}} \times(n-k)\right] .
\end{aligned}
$$

Therefore, the integrity packet duration $t_{\text {packet }}$ with MAC payload length as $N_{\text {MACFrameBody }}$ in bit can be determined by the Eqs. (6)-(8).

$t_{\text {RemainPacket }}$ is the remaining packet duration where the remain packet is not enough to form an integrity packet. The remaining volume of packet $N_{\text {RemainPacket }}$ is defined as

$N_{\text {RemainPacket }}=\lambda_{i} \times t_{\text {superframe }}-($ num -1$) \times N_{\text {MACFrameBody }}$

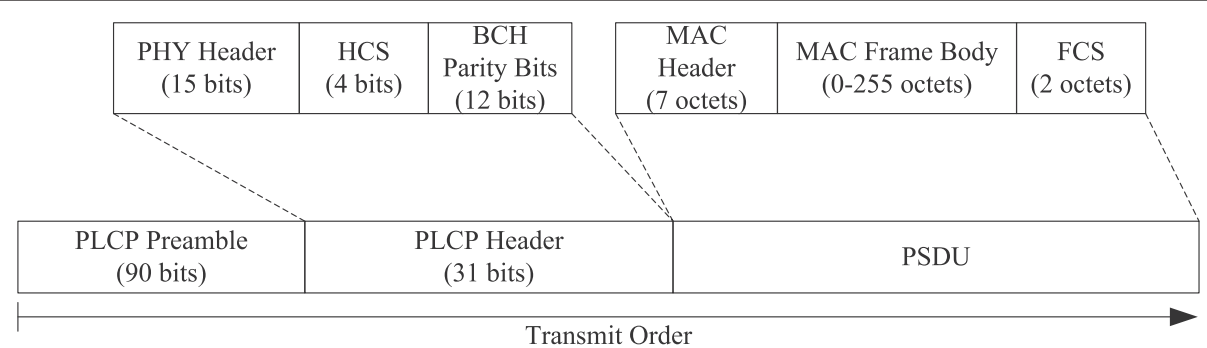

Fig. 10 Physical layer frame structure in IEEE 802.15.6 standard 
then, the remaining packet duration is calculated by Eqs. (6) $-(8)$ with $N_{\text {MACFrameBody }}=N_{\text {RemainPacket }}$.

pSIFS denotes the interframe spacing when packets are transmitted in MAC layer. It can be determined by the receive-to-transmit or transmit-to-receive turnaround time, which is defined as a constant value in IEEE 802.15.6 PHY.

$t_{\mathrm{ACK}}$ is the ACK packet duration which can be deduced through making the $N_{\text {MACFrameBody }}=N_{\text {ACK }}$ in Eqs. (6)-(8).

GT represents the guard time. It guarantees that there is no overlap between the adjacent allocation intervals. IEEE 802.15.6 standard provides either distributed or centralized guard time for a node. Here, we choose the centralized guard time where all allocation intervals are controlled by the hub.

$\mathrm{GT}=\mathrm{GT}_{0}=\mathrm{pSIFS}+\mathrm{pExtraIFS}+m$ ClockResolution,

where pExtraIFS denotes the synchronization error tolerance and $m$ ClockResolution denotes the timing uncertainty.

Due to the IEEE 802.15.6 that is applied in a short range of the human body, we ignore the propagation delay $t_{\text {ProDelay }}$ caused by wireless transmission, which means the $t_{\text {ProDelay }}=0$.

\subsubsection{Number of allocation slots}

In accordance with IEEE 802.15.6, the hub still needs to calculate the number of allocation slots for a node due to the allocation interval in scheduled allocation should be measured by the allocation slot number.

$$
N_{\text {TheoAllocationSlot }}=\left\lceil\frac{t_{\text {interval }}}{\text { AllocationSlotLength }}\right\rceil
$$

However, it should be noticed that in realistic WBAN environment, the wireless link is not as idle as we expected. Therefore, the realistic number of allocation slots should be extended based on the practical channel condition

$$
N_{\text {AllocationSlot }}=U \times N_{\text {TheoAllocationSlot }} \text {, }
$$

where $U$ denotes the scaling up factor which means the number of allocation slots can be extended with several times. The $U$ should be determined by considering the channel condition, interference from other nodes, and delay of hardware processing.

With Eqs. (3)-(14), the relationship between allocation slot number $N_{\text {Allocationslot }}$ and the data arrival rate $\lambda_{i}$ can be determined uniquely, which will guide the hub to assign appropriate number of allocation slots for nodes.

\section{Bit sequence mechanism for $\boldsymbol{m}$-periodic data}

For the routine monitoring, several $m$-periodic sensor nodes exist in the network with heterogeneous data arrival rates. If they are assigned into a superframe, the traffic distribution is unbalanced, which leads to a deterioration in network performance. An example of allocation assignments of $m$-periodic nodes is presented in Fig. 11 . The 2-periodic data and 4-periodic data need two beacon periods and four beacon periods to complete their transmission, respectively. Their data transmissions begin in a same superframe with different slot numbers in Fig. 11a. The occupied slots in the current superframe may be empty in the next superframe or the next few superframes for $m$-periodic data. This is extravagant in WBAN for human monitoring. This kind of allocation for $m$-periodic data results in low resource utility efficiency. On the contrary, the balanced allocation assignment in Fig. 11b possesses a high resource utility efficiency. The slots with same number in different superframes can be assigned to different nodes. The superframe can accommodate more nodes, which highly expands the network capacity. The traffic distribution is balanced at the same time.

To address the unbalanced resource of $m$-periodic data, we introduce a bit sequence-scheduled algorithm to allow different nodes with $m$-periodic data share the same slot number in different surperframes to expand the network capacity. A bit sequence is used to express the transmission condition of $m$-period data in a series of superframes. Different bits express whether the node transmits data

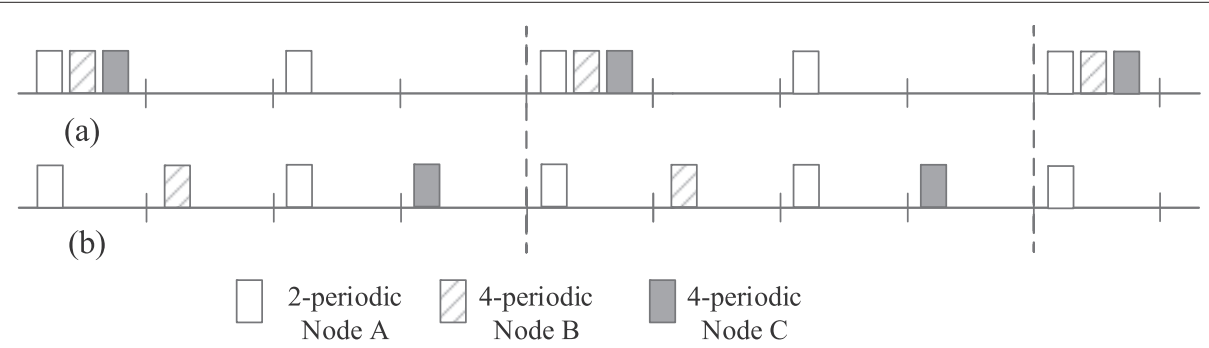

Fig. 11 a An example of unbalanced allocation assignment for $m$-periodic nodes. b An example of balanced allocation assignment for $m$-periodic nodes 
in the current superframe. If $m$-periodic nodes transmit data in a superframe, the sequence bit of a superframe is expressed by "1." If not, by "0." For example, 1-periodic data can be presented as "111111..." 2-periodic data can be presented with a bit sequence "10101010..." or "01010101...," while 4-periodic data have four expressions of bit sequence "100010001000...," "010001000100...," "001000100010...," and "000100010001...."

Considering the cycle property of periodic bit sequence, the sequence length of $m$-periodic data is selected as $l$, which represents the period of generated data. The sequence length of several $m$-periodic data is the least common multiple of them. It should be noticed that the $0-1$-bit sequence is a representation that is different from the binary system. The number " 0 " or " 1 " is a indication whether the $m$-periodic data are transmitted in the superframe.

\subsection{Sequence function}

The bit sequence function is based on the logical operators, which is in correspondence with the bit sequence. In our bit sequence-scheduled algorithm, only logical multiplication and logical addition are used, i.e., "AND" operation and "OR" operation. The bit sequence functions of several nodes with different $m$ periods can be expressed as Eqs. (17) and (18) based on Eqs. (15) and (16). The parameter $k$ is the least common multiple of the $m$-periodic nodes while $i$ indicates the bit in the sequence.

$$
\begin{aligned}
\operatorname{AND}(i, j) & = \begin{cases}1, & \text { if } i=1, j=1 \\
0, & \text { else }\end{cases} \\
\operatorname{OR}(i, j) & = \begin{cases}0, & \text { if } i=0, j=0 \\
1, & \text { else }\end{cases} \\
A\left(s_{1}, s_{2}, \cdots, s_{n}\right) & =\sum_{i=1}^{k} \operatorname{AND}\left(s_{1}[i], s_{2}[i], \cdots, s_{n}[i]\right)( \\
O\left(s_{1}, s_{2}, \cdots, s_{n}\right) & =\sum_{i=1}^{k} \mathrm{OR}\left(s_{1}[i], s_{2}[i], \cdots, s_{n}[i]\right)
\end{aligned}
$$

The "AND" function or the logical multiplication function is applied to evaluate whether the nodes are mutually exclusive on the slot sharing in different superframes with the same slot number. If the results of logical multiplication are all zero, it indicates that the nodes can share the same slot number in different superframes without collision. If there is " 1 " value in the logical multiplication sequence, it means the slots with the same number in different superframes are utilized and that different nodes cannot share the slot with same number. The "OR" function or logical addition function is employed to express whether they can provide available slots for extra nodes. The zero value in the result of "OR" function indicates that the slot in different superframes is unoccupied and can be assigned to other nodes for data transmission. If the results of logical multiplication are all "1", the slots in different beacon periods are fully occupied in the highest utility that cannot support slot allocation to new admitted

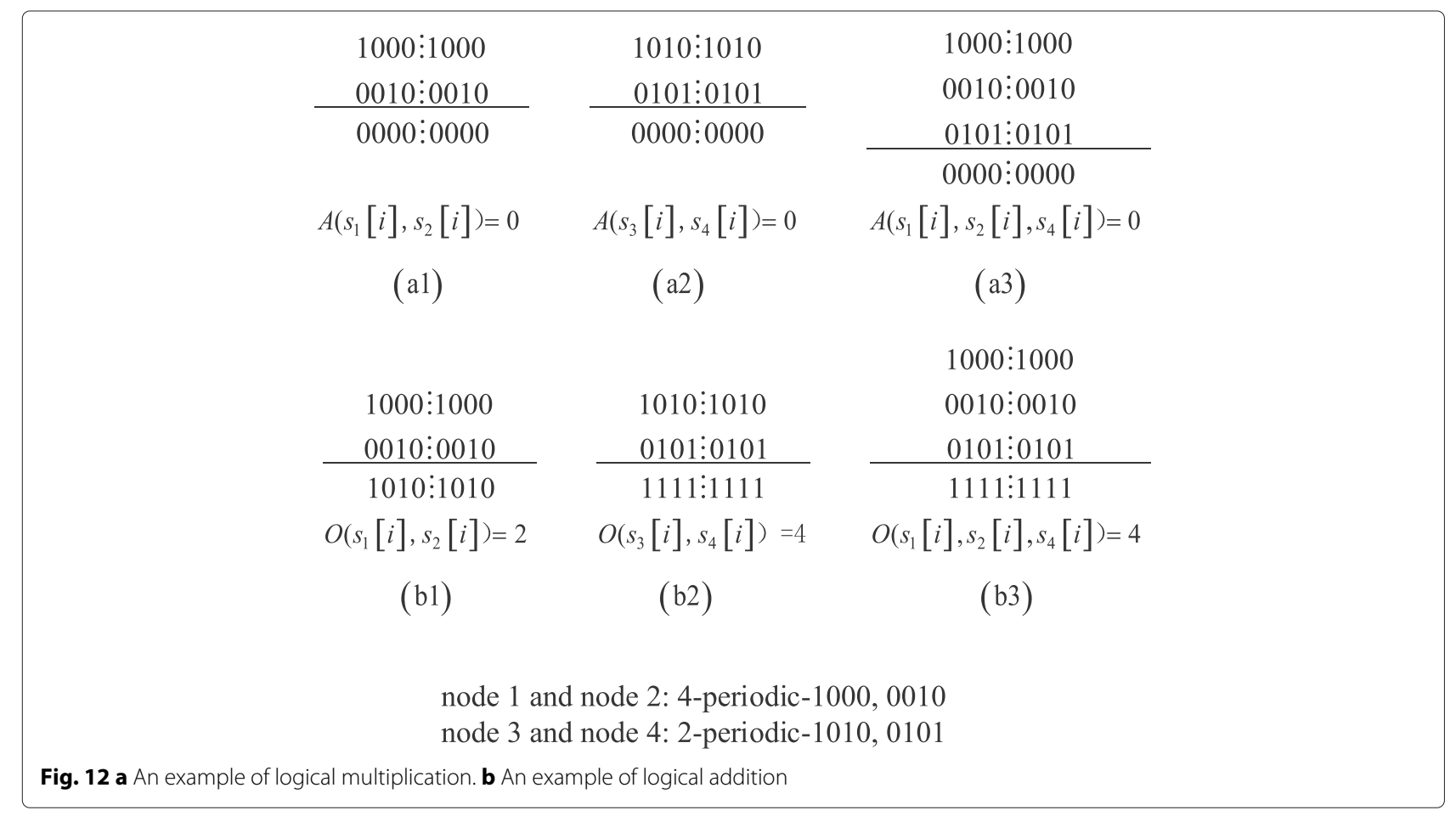


nodes. But there are other slots with different slot number that can be provided to the new admitted nodes.

An example of 2-periodic and 4-periodic bit sequences in "AND" and "OR" operation is illustrated in Fig. 12. The sequence lengths of 2-periodic and 4-periodic data are different. In Fig. 12 (a1, a2), the "AND" function result is zero, so node 1 and node 2 can share the same slot number while node 3 and node 4 share the slot number in different superframes. Considering the "OR" function result in Fig. 12 (b2), the slots are fully utilized by 2-periodic data since the value of "OR" function is equal to the sequence length. However, in Fig. 12 (b1), there are available slots for one more 2-periodic node except for node 1 and node 2. At the same time, the results in (a3) and (b3) validate the scheme. Two 4-periodic nodes and one 2-periodic node share the same slot in different superframes. The "AND" and "OR" operation satisfy the commutation law and association law. Whether multiple nodes with different $m$ periods can share the slots resource depends on the values of the bit sequence function.

\subsection{Bit sequence-scheduled algorithm}

The specific bit sequence-scheduled algorithm is presented as follows. The network capacity can be largely expanded owing to the full utility of every slot in different superframes. Node $i$ and node $j$ can make a judgement if they can share the slots with same number in different superframes through the "AND" and "OR" function. Several nodes that share the slots may operate the algorithm repeatedly to realize the highest utilization of slots and the network capacity expansion. The nodes having common factors except for 1 are in high possibility to share the slots and reduce the waste of slots resource. Node $j$ cyclic

Table 2 Simulation parameters

\begin{tabular}{ll}
\hline Parameters & Value \\
\hline pAllocationSlotMin & $500 \mu \mathrm{s}$ \\
pAllocationResolution & $500 \mu \mathrm{s}$ \\
pSIFS & $75 \mu \mathrm{s}$ \\
pExtralFS & $10 \mu \mathrm{s}$ \\
mClockResolution & $4 \mu \mathrm{s}$ \\
Code rate $(k n)$ & $51 / 63$ \\
PSDU spreading factor & 1 \\
Symbol rate $(k s p s)$ & 600 \\
PLCP header spreading factor & 4 \\
Voltage supply & $3 \mathrm{~V}$ \\
Transmitted current $\left(l_{\text {tx }}\right)$ & $7.5 \mathrm{~mA}$ \\
Received current $\left(I_{\text {rx }}\right)$ & $13.1 \mathrm{~mA}$ \\
Standby current $\left(l_{\text {idle }}\right)$ & $26 \mu \mathrm{s}$ \\
Sleep current $\left(I_{\text {sleep }}\right)$ & $900 \mathrm{nA}$ \\
\hline
\end{tabular}

shifts the bit sequence since there are several expressions for $m$-periodic data $(m>1)$.

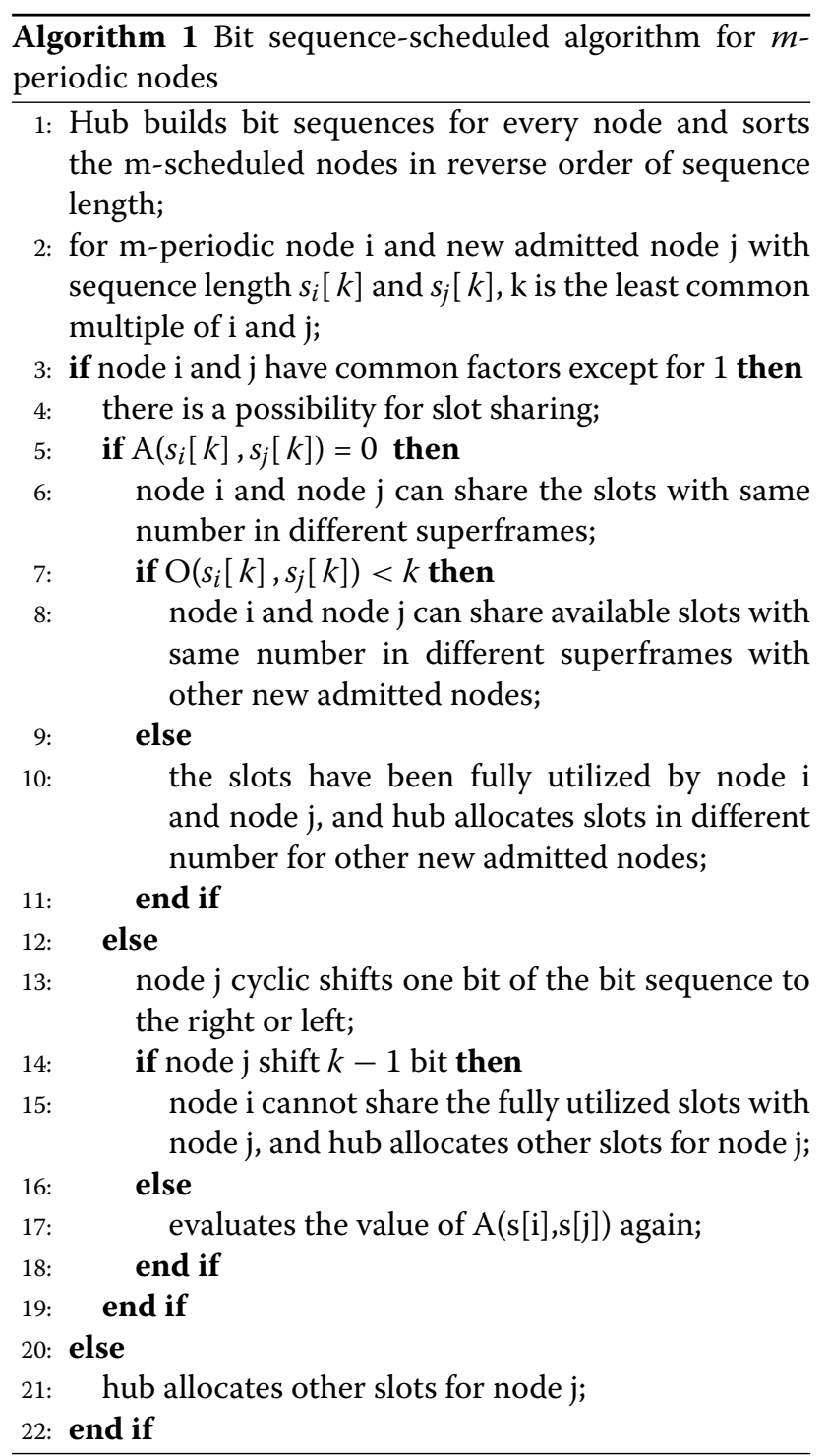

In general, $m$-periodic data are in relatively lower user priority. They are mostly used for long-term monitoring. Through the bit sequence-scheduled algorithm, the heavy network traffic of different nodes is assigned to several superframes. The resource in the WBAN can be fully utilized, and more nodes can be admitted to the network for data transmission. At the same time, the network traffic transactions become more balanced for $m$-periodic sensor nodes.

\section{Performance evaluation}

In order to examine the effectiveness of the proposed TTR MAC, we conduct the TTR MAC in MATLAB and compare the simulation results with the two ubiquitous MAC 
protocols. One protocol is the IEEE 802.15.6, where the hub assigns fixed allocation slot number for each sensor node without considering the heterogenous data arrival rates. Nodes will contend for the channel in CSMA/CA if the allocated slots are insufficient to transmit their data. The other protocol represents a kind of protocol which is based on one-round reservation to adapt the diverse data arrival rates, named OR MAC here. In the OR MAC, the hub first assigns a fixed slot for each node to inform its data volume. Once collecting all information about buffered data from all nodes, the hub assigns allocation slots for each node based on the collected information.

\subsection{Simulation parameters}

Simulation parameters complied with IEEE 802.15.6 specifications are shown in Table 2. The current values are derived from [39]. In addition, there are also some parameters are self-defined. A superframe consists of 255 slots, and the payload of each packet is 255 bytes. The analysis is based on the ideal channel condition. The effect of bit errors in the channel is neglected. In other words, a packet is dropped only due to packet collision or device buffer overflow. Besides, we make the scaling up factor $U$ as 1 that the allocation assignment satisfies the demands of data transmission exactly.

\subsection{Energy analysis}

We consider the scenario that there are eight nodes and a hub in the network, each node generates periodic data with data arrival rate varying from 1 to $8 \mathrm{kbps}$. Nodes are numbered with No.1, No.2,.., No.8 in accordance with their data arrival rate. Burst data generate when the collected data are out of the safe range.

\subsubsection{Energy efficiency}

First, we evaluate the network performance when the number of nodes with burst data increases. The energy efficiency of the whole network is defined as the ratio that the energy caused by transmitting sensor data from eight nodes to total energy consumption.

Figure 13 shows the comparison of the energy efficiency between TTR MAC and the other two MAC protocols. The TTR MAC always outperforms the other two MACs while the number of nodes with burst data increases. Compared with IEEE 802.15.6, TTR MAC assigns the appropriate allocation slot number for each node which utilizes the channel effectively. What's more, nodes in IEEE 802.15.6 will contend for the channel in CSMA/CA to transmit data when allocation slots are insufficient, which will cost additional energy in idle listening, overhearing, and collision with each other. Compared with OR MAC, TTR MAC raises the first-round reservation once the network starts with nearly no overhead and raises the second-round reservation when the nodes have burst data to transmit. Nodes in TTR MAC cost less energy to transmit the control information about the allocation request frames while OR MAC will transmit all the allocation request frames from each node in every superframe without considering whether the node has burst data or not. The energy efficiency of TTR MAC and IEEE 802.15.6 decreases with the increasing number

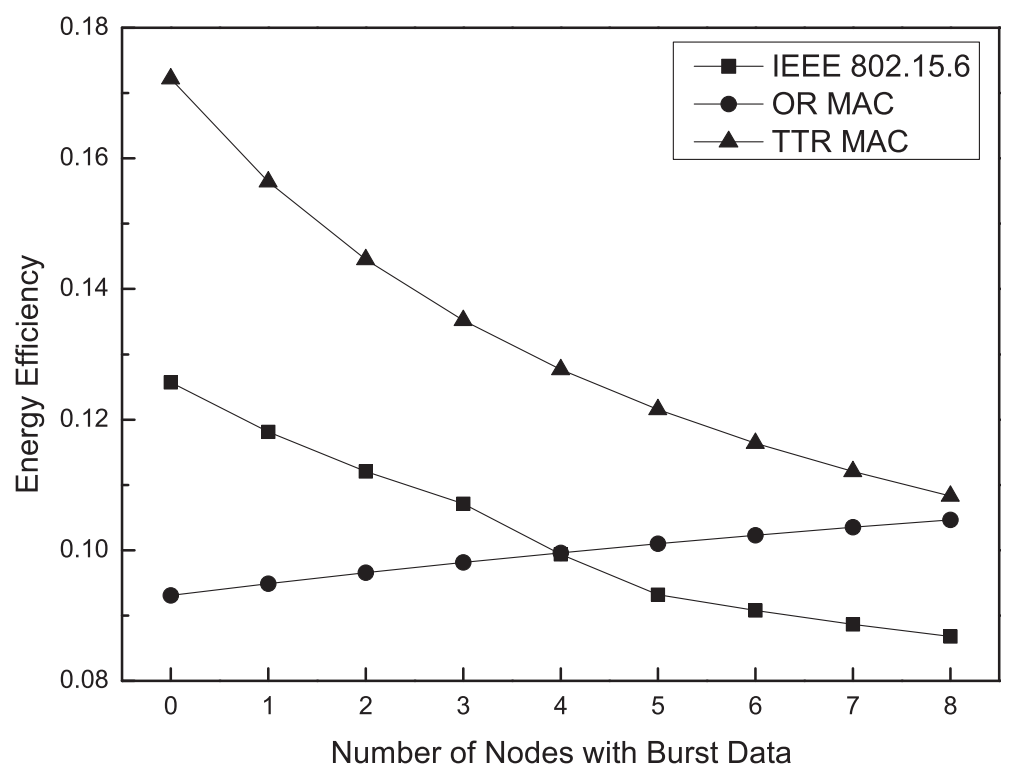

Fig. 13 Energy efficiency versus the number of nodes with burst data 


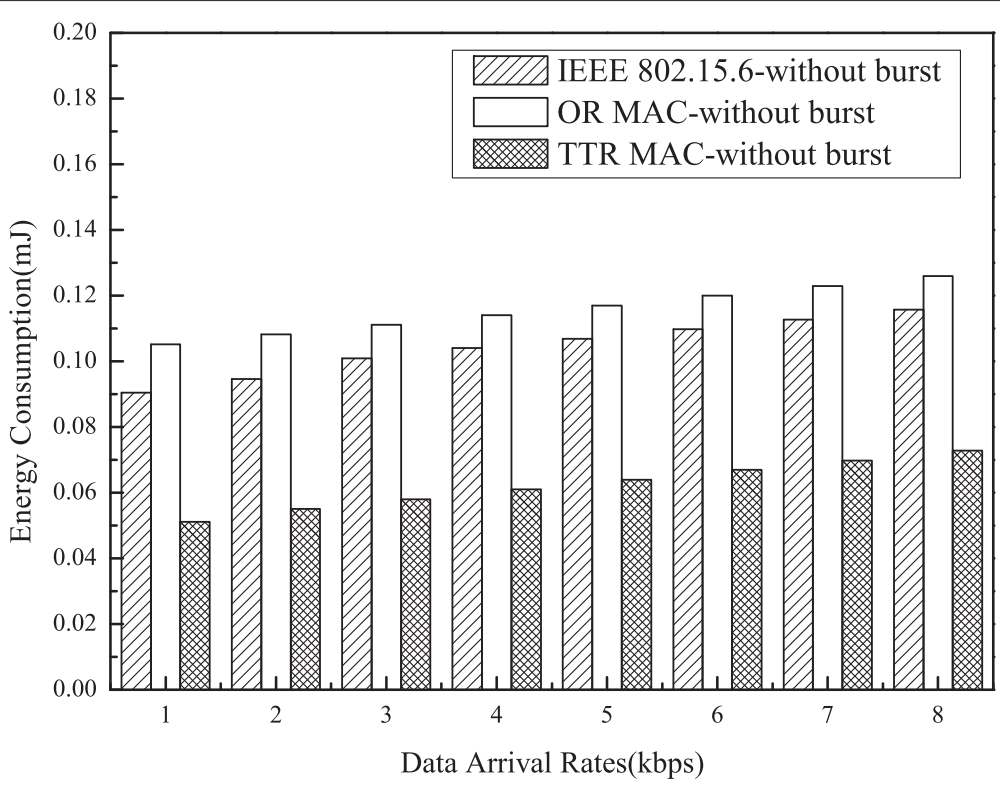

Fig. 14 Energy consumption with periodic data versus data arrival rates

of nodes with burst data, since the IEEE 802.15.6 will suffer more frequent packet collisions and TTR MAC will generate more additional control packets when burst data arise. In contrast, the energy efficiency of OR MAC increases due to that it reserves the time of control packets for each node in each superframe. In numerical results, TTR MAC can improve the energy efficiency up to 84.97 and $36.96 \%$ compared with OR MAC and IEEE 802.15.6, respectively, once there is no burst data in the network.

\subsubsection{Energy consumption}

Energy consumption $E_{\text {state }}$ is a function of time and current drawing from voltage source for a specific state, which is shown in Eq. (19). Here, we utilize the coin cell batteries that provide constant operating voltage during their lifetime, which means the $V_{\text {state }}$ is a constant value. The current $I_{\text {state }}$ in specific states are shown in Table 2, including $I_{\mathrm{tx}}, I_{\mathrm{rx}}, I_{\mathrm{idle}}$, and $I_{\text {sleep }}$. The $t_{\mathrm{state}}$ denotes the time cost in specific state, which includes transmitted, received, idle, and sleep state.

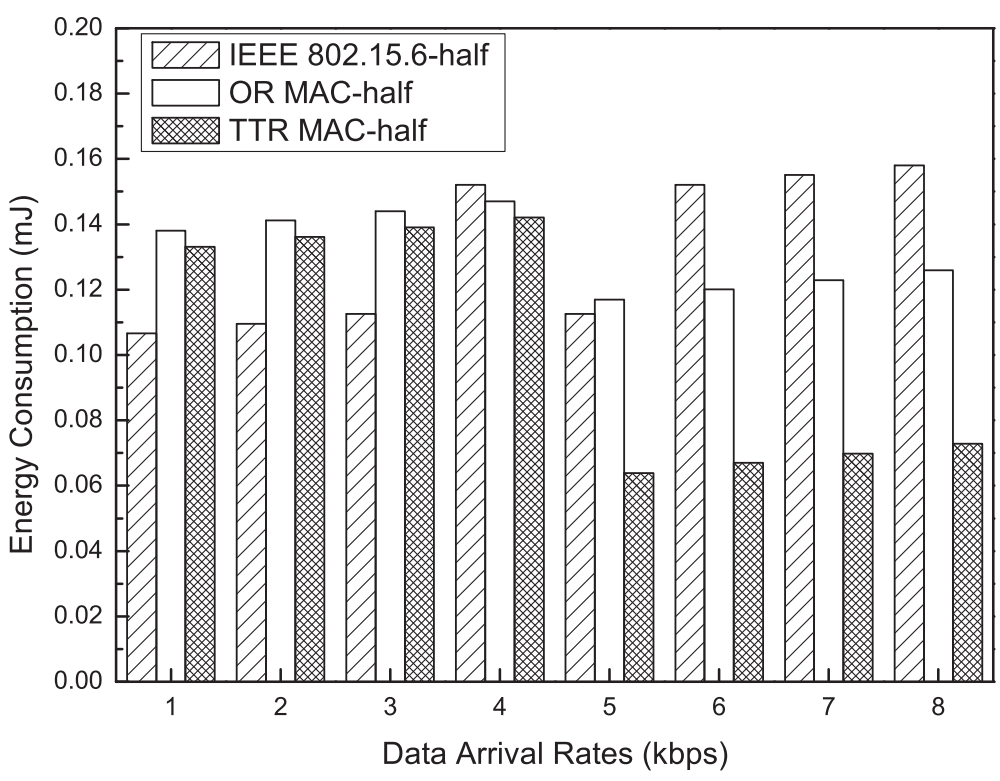

Fig. 15 Energy consumption versus data arrival rates 


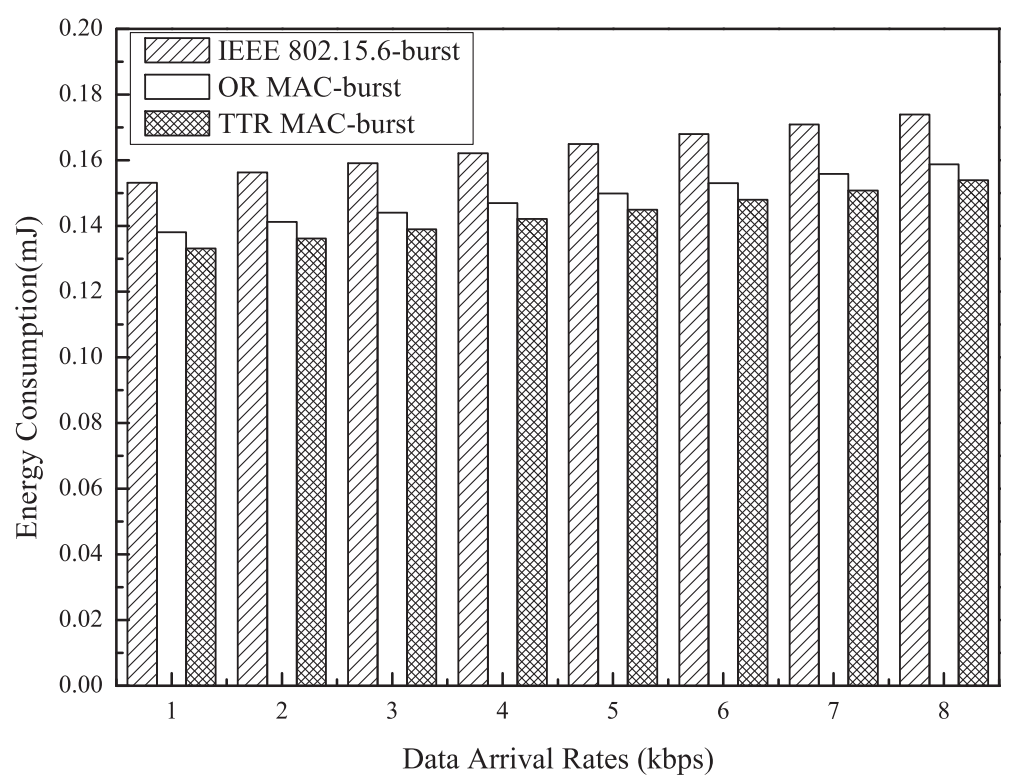

Fig. 16 Energy consumption with burst data versus data arrival rates

$$
\begin{aligned}
E_{\text {state }} & =V_{\text {state }} \times I_{\text {state }} \times t_{\text {state }} \\
T_{\mathrm{tx}} & =T_{\text {AllocRequset }}+T_{\text {data }} \\
T_{\mathrm{rx}} & =T_{\text {AllocAssignment }}+T_{\text {Beacon }}+T_{\mathrm{ACK}} \\
T_{\text {idle }} & =2 \mathrm{pSIFS}+\mathrm{GT}+\text { delay } \\
T_{\text {sleep }} & =m \times \text { superframe }-T_{\mathrm{tx}}-T_{\mathrm{rx}}-T_{\text {idle }},
\end{aligned}
$$

where $T_{\mathrm{tx}}$ is the time cost in transmitted state, including the time needed to transmit reservation request frames and data frames. $T_{\mathrm{rx}}$ is the time consumed in received state, including the time needed to transmit reservation assignment frames, beacon frames, and ACK frames. $T_{\text {idle }}$ consists of the receive-to-transmit or transmit-to-receive turnaround time, guard time, and propagation delay time. Nodes turn into sleep state when there is no data transmission. $T_{\text {sleep }}$ is the time lasted in sleep state.

The energy consumption of each node in a superframe is shown in Figs. 14, 15, and 16. There is no burst data in the network in Fig. 14. Only nodes 1-4 have burst data in Fig. 15 while every node has burst data in Fig. 16. The

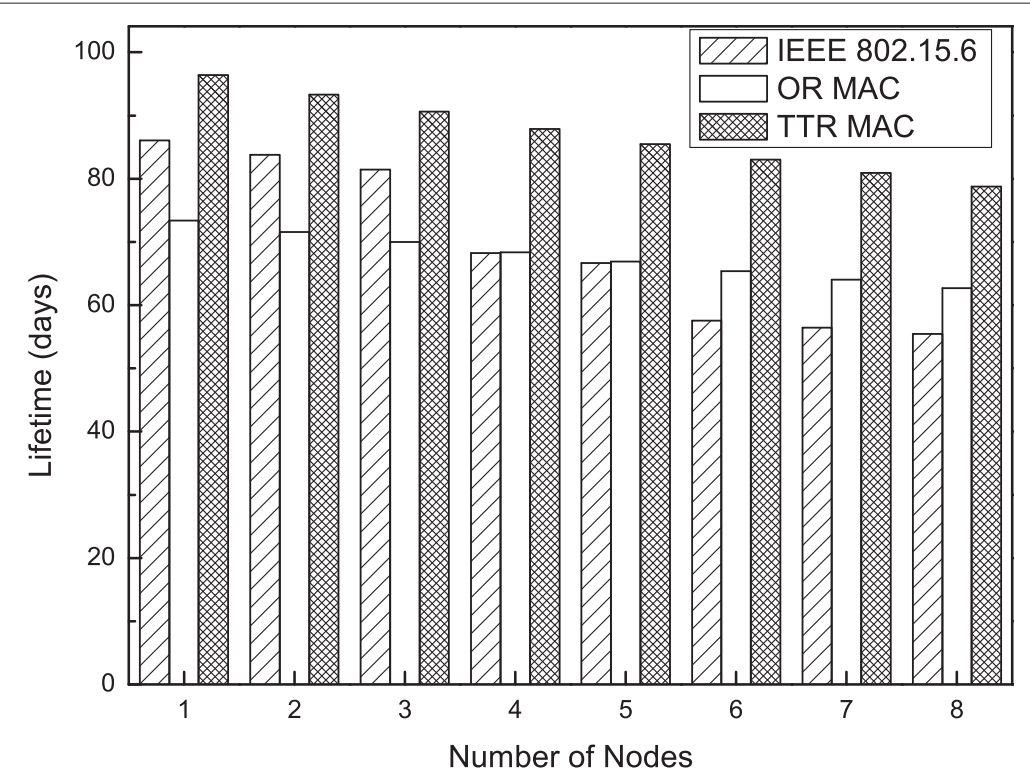

Fig. 17 Lifetime versus number of nodes 
higher data arrival rate is, the more energy nodes will consume. For nodes with light traffic, IEEE 802.15.6 consumes less energy than OR MAC since the node can access to channel fast with no overhead. As the traffic increases, IEEE 802.15.6 will consume more energy due to the frequent packet collisions. Besides, the burst data increase the probability of collision in IEEE 802.15.6, which consumes mores energy than the other two MACs, as shown in Fig. 16. TTR MAC reduces energy consumption greatly both for the nodes with burst data and without burst data, because TTR MAC has handled the periodic data well in the first-round reservation with little overhead and employs the second-round reservation for burst data.

\subsubsection{Lifetime}

Sensor nodes in the health monitoring application should live as long as possible due to the limitation to recharge the nodes, especially for the implanted sensor nodes. Thus, we evaluate the lifetime of sensor nodes with different data arrival rates in health monitoring. Here, we assume that the eight nodes are equipped with button cell of capacity $Q \mathrm{mAh}$, which is a constant value as $560 \mathrm{mAh}$. The lifetime of the sensor nodes can be calculated as Eq. (24).

$$
\text { lifetime }=\frac{Q}{E_{\text {node }}} \times t_{\text {superframe }}
$$

The $E_{\text {node }}$ denotes the average energy consumption of each node in a superframe. Assuming that a node generates burst data with equal probability, $E_{\text {node }}$ can be obtained from Eq. (25).

$$
E_{\text {node }}=\frac{E_{\text {WithoutBurst }}+E_{\text {WithBurst }}}{2}
$$

Figure 17 shows the lifetime of nodes with variable data arrival rates. The higher the data arrival rate is, the shorter lifetime is. Because nodes with higher data arrival rate need more energy to transmit their data. By raising tworound reservations to handle the periodic data and burst data specifically, TTR MAC can save more energy than OR MAC and IEEE 802.15.6. The maximum lifetime for nodes with $1 \mathrm{kbps}$ in TTR MAC, OR MAC, and IEEE 802.15.6 are $96.40,73.39$, and 86.06 days, respectively. In other words, TTR MAC prolongs the lifetime of nodes with 31.35 and $12.01 \%$ compared with OR MAC and IEEE 802.15.6.

Furthermore, the relationship between nodes in different $m$-periods and the lifetime of sensor nodes is presented in Fig. 18. It is obvious that the lifetime of all three MAC protocols is increasing with the $m$ in higher value, since the higher value of $m$ indicates that the node has a lower duty cycle and sleeps longer period with minimum energy consumption. Moreover, nodes in the network generate periodic data with different data arrival rates. The lifetime of nodes with data arrival rate $2 \mathrm{kbps}$ is longer than that of $7 \mathrm{kbps}$, which is attributed to the high traffic that consumes more energy and suffers shorter lifetime. At the same time, the probability of the generated burst data in the network increases with the increasing data arrival rate, and the average energy consumption increases accordingly. But the TTR MAC still performs better than IEEE 802.15.6 and OR MAC with heterogenous data arrival rates in the WBAN owing to the

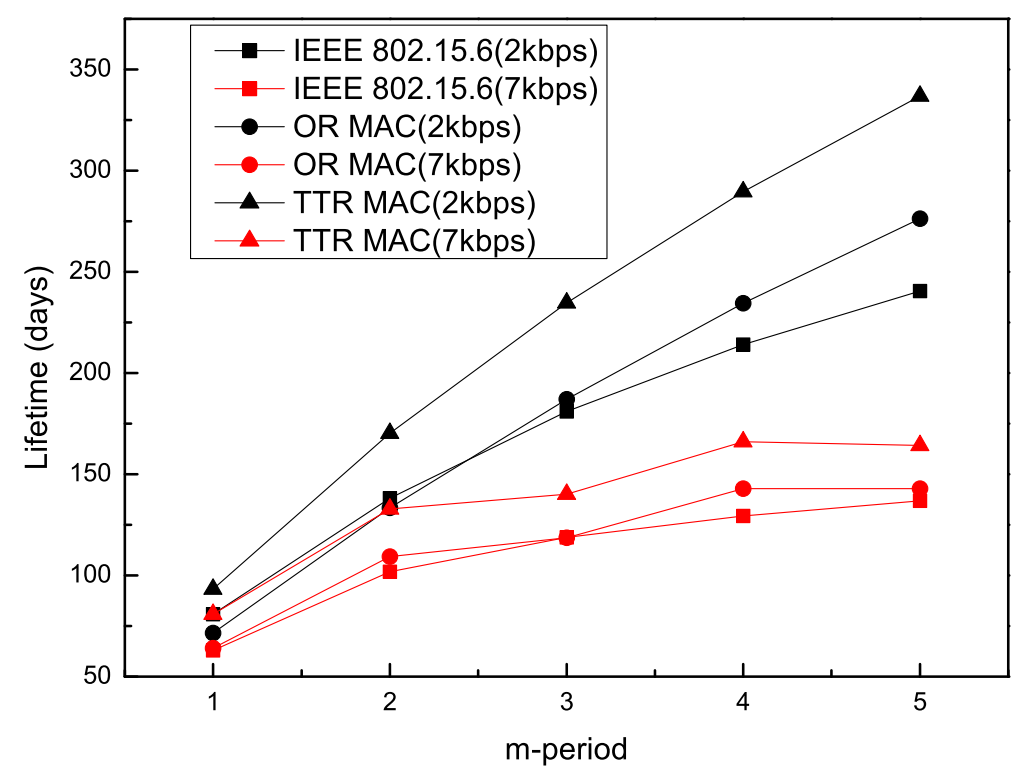

Fig. 18 Lifetime versus nodes in different $m$-periods 
two reservation modes that generate low control packets overhead.

\section{Conclusions}

In this paper, we propose a reliable and energy-efficient MAC protocol named TTR MAC for health monitoring. TTR MAC raises two kinds of reservation modes for periodic data and burst data, which becomes more adaptive and reduces the energy consumption simultaneously. Besides, the introduced token parameter discriminates the transmission order of correlated burst data, providing better quality of service for the urgent data. Moreover, a bit sequence-scheduled algorithm for $m$-periodic data is designed to increase the resource utility and network capacity. The analysis about TTR MAC is conducted, and the simulation results show that the energy efficiency of TTR MAC achieves up to 84.97 and $36.96 \%$ improvement than OR MAC and IEEE 802.15.6, respectively. Simultaneously, TTR MAC prolongs the lifetime of sensor nodes up to 31.35 and $12.01 \%$. Our future work will focus on determining the optimal parameters in TTR MAC to adapt the complex channel conditions in health monitoring, for example, designing the adaptive scaling up factor $U$ in TTR MAC under different channel conditions. The performance evaluation of the bit sequence-scheduled algorithm will be executed as well.

\section{Competing interests}

The authors declare that they have no competing interests.

\section{Acknowledgements}

This work was supported by the National Natural Science Foundation of China under Grant No. 61271176, No. 61401334, and No. 61571350, the National Science and Technology Major Project under Grant No. 2013ZX03005007-003, the Fundamental Research Funds for the Central Universities (BDY021403, JB140113), and the 111 Project (B08038).

\section{Author details}

${ }^{1}$ State Key Laboratory of Integrated Services Networks, Xidian University, No.2 Tai Bai South Road, 710071 Xi'an, China. ${ }^{2}$ Department of Computer and Software Technology, University of Swat, Odigram, Pakistan. ${ }^{3}$ CISTER Research Center, ISEP, Polytechnic Institute of Porto (IPP), Rua Dr. Antonio Bernardino de Almeida 431, 4249015 Porto, Portugal.

Received: 1 November 2015 Accepted: 24 April 2016

Published online: 04 May 2016

\section{References}

1. RSH Istepanian, S Laxminarayn, CS Pattichis, M-health: Emerging Mobile Health Systems. (R Istepanian, S Laxminarayan, CS Pattichis, eds.) (Springer, Berlin, 2006), pp. 237-246

2. M Kay, J Santos, M Takane, mhealth: new horizons for health through mobile technologies. World Health Organization. 64(7), 66-71 (2011)

3. H Oh, C Rizo, M Enkin, A Jadad, What is eHealth (3): a systematic review of published definitions. J. Med. Internet Res. 7(1), e1 (2005). doi:10.2196/jmir.7.1.e1

4. M Chen, S Gonzalez, A Vasilakos, H Cao, VC Leung, Body area networks: a survey. Mobile Netw. Appl. 16(2), 171-193 (2011)

5. S Ullah, H Higgins, B Braem, B Latre, C Blondia, I Moerman, S Saleem, Z Rahman, KS Kwak, A comprehensive survey of wireless body area networks. J. Med. Syst. 36(3), 1065-1094 (2012)
6. DM Barakah, M Ammad-uddin, in Intelligent Systems, Modelling and Simulation (ISMS), 2012 Third International Conference On. A survey of challenges and applications of wireless body area network (WBAN) and role of a virtual doctor server in existing architecture (IEEE, 2012), pp. 214-219. Kota Kinabalu, Malaysia, 8-10 February 2012

7. H Cao, V Leung, C Chow, H Chan, Enabling technologies for wireless body area networks: a survey and outlook. IEEE Commun. Mag. 47(12), 84-93 (2010)

8. E Kartsakli, AS Lalos, A Antonopoulos, S Tennina, MD Renzo, L Alonso, C Verikoukis, A survey on M2M systems for mHealth: a wireless communications perspective. Sensors. 14(10), 18009-18052 (2014)

9. Latré, B Braem, I Moerman, C Blondia, P Demeester, A survey on wireless body area networks. Wireless Netw. 17(1), 1-18 (2011)

10. A Boulis, D Smith, D Miniutti, L Libman, Y Tselishchev, Challenges in body area networks for healthcare: the MAC. Commun. Mag. IEEE. 50(5), 100-106 (2012)

11. R Cavallari, F Martelli, R Rosini, C Buratti, R Verdone, A survey on wireless body area networks: technologies and design challenges. Commun. Surv. Tutor. IEEE. 16(3), 1635-1657 (2014)

12. $\mathrm{IHa}$, Technologies and research trends in wireless body area networks for healthcare: a systematic literature review. Int. J. Distributed Sensor Netw. 2015(4), 1-14 (2015)

13. $\mathrm{H} \mathrm{Li}, \mathrm{J} \mathrm{Tan}$, in IEEE 27th Annual International Conference of the Engineering in Medicine and Biology Society, IEEE-EMBS. An ultra-low-power medium access control protocol for body sensor network, (2005), pp. 2451-2454. Shanghai, China, 31 August -3 September 2005

14. IEEE 802.15.6 standard, Wireless body area networks. IEEE Computer Society. IEEE Standard. 802.15.6-2012, 1-271(2012)

15. IEEE 802.15.4 standard, Low-rate wireless personal area networks (LR-WPANs). IEEE Computer Society. IEEE Standard. 802, 4-2011 (2011). 802.15.4-2011, 1-294(2011)

16. S Ullah, B Shen, S Riazul Islam, P Khan, S Saleem, K Sup Kwak, A study of MAC protocols for WBANs. Sensors. 10(1), 128-145 (2009)

17. T van Dam, K Langendoen, in Proceedings of the 1st International Conference on Embedded Networked Sensor Systems. An adaptive energy-efficient MAC protocol for wireless sensor networks, (2003), pp. 171-180. Los Angeles, California, USA, November 5-7, 2003

18. A El-Hoiydi, J-D Decotignie, in Computers and Communications, 2004. Proceedings. ISCC 2004. Ninth International Symposium On. WiseMAC: an ultra low power MAC protocol for the downlink of infrastructure wireless sensor networks, vol. 1 (IEEE, 2004), pp. 244-251. Alexandria, Egypt, June 28-July 1, 2004

19. W YE, An energy-efficient MAC protocol for wireless sensor networks. Wireless Sensor Netw. 01(10), 1567-1576 (2009)

20. S Ullah, M Chen, KS Kwak, Throughput and delay analysis of IEEE 802.15. 6-based CSMA/CA protocol. J. Med. Syst. 36(6), 3875-3891 (2012)

21. SRashwand, J Misic, V Misic, Analysis of CSMA/CA mechanism of IEEE 802.15. 6 under non-saturation regime. IEEE Trans. Parallel and Distributed Systems. 27(5), 1279-1288 (2015)

22. S Rashwand, J Mišić, H Khazaei, IEEE 802.15. 6 under saturation: some problems to be expected. Commun. Netw. J. 13(2), 142-148 (2011)

23. V Esteves, A Antonopoulos, E Kartsakli, M Puig-Vidal, P Miribel-Català, C Verikoukis, Cooperative energy harvesting-adaptive MAC protocol for WBANs. Sensors. 15(6), 12635-12650 (2015)

24. PK Tiwary, W-S Baek, D-M Kim, J-Y Pyun, in TENCON 2012-2012 IEEE Region 10 Conference. A study on ultra low power MAC protocols over wireless body area network (IEEE, 2012), pp. 1-6. Cebu, Philippines, 19-22 November 2012

25. S Ullah, KS Kwak, An ultra low-power and traffic-adaptive medium access control protocol for wireless body area network. J. Med. Syst. 36(3), 1021-1030 (2012)

26. M Al Ameen, N Ullah, MS Chowdhury, SR Islam, K Kwak, A power efficient MAC protocol for wireless body area networks. EURASIP J. Wireless Commun. Network. 2012(1), 1-17 (2012)

27. Z Nie, Z Li, R Huang, Y Liu, J Li, L Wang, A statistical frame based TDMA protocol for human body communication. Biomedical Eng. Online. 14(1), 65 (2015)

28. E Ibarra, A Antonopoulos, E Kartsakli, JJ Rodrigues, C Verikoukis, in Communications (ICC), 2014 IEEE International Conference On. Joint power-QoS control scheme for energy harvesting body sensor nodes, (IEEE, 2014), pp. 3511-3516. Sydney, Australia, 16-20 June 2014 
29. E Ibarra, A Antonopoulos, E Kartsakli, JJ Rodrigues, C Veri koukis, QoS-aware energy management in body sensor nodes powered by human energy harvesting. Sensors J. IEEE. 16(2), 542-549 (2016)

30. E Kartsakli, A Antonopoulos, L Alonso, C Verikoukis, A cloud-assisted random linear network coding medium access control protocol for healthcare applications. Sensors. 14(3), 4806-4830 (2014)

31. Marinković, EM Popovici, C Spagnol, S Faul, WP Marnane, Energy-efficient low duty cycle MAC protocol for wireless body area networks. Information Technol. Biomedicine, IEEE Trans. 13(6), 915-925 (2009)

32. MM Alam, O Berder, D Menard, O Sentieys, TAD-MAC: traffic-aware dynamic MAC protocol for wireless body area sensor networks. IEEE J. Emerging Selected Topics Circuits Syst. 2(1), 109-119 (2012)

33. Z Li, M Chen, G Zhang, Variable-rate transmission method with coordinator election for wireless body area networks. Wireless Networks. 21(7), 1-12 (2015)

34. G Fang, E Dutkiewicz, in Communications and Information Technology, 2009. ISCIT 2009. 9th International Symposium On. BodyMAC: Energy efficient TDMA-based MAC protocol for wireless body area networks (IEEE, 2009), pp. 1455-1459. Incheon, Korea, 28-30 September 2009

35. C Li, B Hao, K Zhang, Y Liu, J Li, A novel medium access control protocol with low delay and traffic adaptivity for wireless body area networks. J. Med. Syst. 35(5), 1265-1275 (2011)

36. B Pourmohseni, M Eshghi, Reliable energy-efficient dynamic-TDMA MAC protocol for wireless body area networks. Int. J. Appl. Innovation Eng. Manag.(IJAIEM). 2(7), 393-402 (2013)

37. O Gama, R Simoes, A hybrid MAC scheme to improve the transmission performance in body sensor networks. Wireless Pers. Commun. 80(3), 1263-1279 (2015)

38. E Ibarra, A Antonopoulos, E Kartsakli, C Verikoukis, HEH-BMAC: hybrid polling MAC protocol for WBANs operated by human energy harvesting. Telecommun. Syst. 58(2), 111-124 (2015)

39. C Tachtatzis, F Di Franco, DC Tracey, NF Timmons, J Morrison, in IEEE GLOBECOM Workshops (GC Wkshps). An energy analysis of IEEE 802.15.6 scheduled access modes, (2010), pp. 1270-1275. Miami, Florida, USA, 6-10 December 2010

\section{Submit your manuscript to a SpringerOpen ${ }^{\odot}$ journal and benefit from:}

- Convenient online submission

- Rigorous peer review

- Immediate publication on acceptance

- Open access: articles freely available online

- High visibility within the field

- Retaining the copyright to your article

Submit your next manuscript at $\mathbf{s p r i n g e r o p e n . c o m ~}$ 